\title{
Prescription practice in patients of upper respiratory tract infection at a pediatric outpatient clinic in Punjab
}

\author{
Pramil Tiwari, ${ }^{1}{ }^{\text {Rajiv Ahlawat }}{ }^{1}$ and Gaurav Gupta ${ }^{2}$ \\ ${ }^{1}$ Department of Pharmacy Practice, National Institute of Pharmaceutical Education and Research (NIPER), Sector 67, S.A.S. Nagar, \\ Punjab-160062, INDIA \\ ${ }^{2}$ Consultant paediatrician, Charak Care Clinics, Phase 7, S.A.S. Nagar, Punjab-160055, INDIA
}

\begin{abstract}
Upper respiratory tract infections (URTI) are the most common and frequently occurring infections in the paediatric population. Often, it has a viral origin and requires only symptomatic treatment. Very few patients require antibiotics. The prescriptions of children, diagnosed with URTI, over one year period of time, were studied to assess the utilization of drugs. Of 2981 patients, only 1078 patients were diagnosed to have URTI. This consisted of 598 male \& 480 female patients. It was found that URTI was most common in the age group of $12-60$ months $(51 \%)$ followed by 1 day-12 months $(20 \%)$. URTI was found to be affect the patients mostly in winter $(35 \%)$ followed by autumn $(25 \%)$. Cough and cold combinations were the most commonly prescribed medicines in URTI ( $27 \%$ of total drugs prescribed) followed by antipyretics $(21 \%)$, herbal cough and cold medicine $(18 \%)$, saline nasal drops $(15 \%)$, bronchodilators $(8 \%)$, antihistamine $(6 \%)$ and antibiotics $(5 \%)$. It was found that $12 \%$ of the patients were prescribed an antibiotic. Amongst antibiotics, azithromycin was the most commonly prescribed followed by amoxicillin + clavulanic acid combination ( $61 \%$ and $31 \%)$. In this study, children less than 5 years of age were the ones diagnosed with URTI and this was highest during the winter season. Azithromycin was most commonly prescribed antibiotic. The antibiotic usage pattern reflects judicious choice of the prescriber.
\end{abstract}

Keywords: Children, Pediatrics, Upper respiratory tract infection, Drug utilization.

\section{INTRODUCTION}

Upper respiratory tract infections (URTIs) include common cold, laryngitis, pharyngitis/tonsillitis, acute rhinitis, acute rhinosinusitis and acute otitis media. ${ }^{1}$ URTIs are believed to be one of the main reasons for visit to the pediatrician and are considered to be the most common reason for the absenteeism from school. The most common signs \& symptoms observed in URTI are coughing and sneezing, congestion, runny nose, low-grade fever, anorexia and myalgia. ${ }^{2}$

Most of the time URTIs have a viral origin; sometimes, they may be due to bacterial infection. The major challenge for clinicians, therefore, is to differentiate between viral and bacterial origin of the URTI. Accurate judgment of origin of infection assists prescriber to prescribe rationally. Rational use of drug aims to minimize the drug-related adverse events, development of resistant strains of microorganism, patient noncompliance and increased cost of treatment. In patients diagnosed with URTI, the first choice is Amoxicillin+clavulanate; and Ceftriaxone is the choice for patients sensitive to penicillin. Other antibiotic used for URTI treatment could include azithromycin, cefuroxime, cefpodoxime, cefexime, cefdinir, cefodroxil. ${ }^{3}$

The management of URTI of viral origin involves providing the symptomatic relief only. For relief of fever, nasal congestion and coughing in viral URTI, a large variety of preparations are available. These com-
Submitted Date : 04-04-2014 Accepted Date : 23-05-2014

DOI: 10.5530/ijopp.7.2.6

Address for correspondence: Pramil Tiwari, M. Pharm., PhD.

Department of Pharmacy Practice, National Institute of Pharmaceutical Education and Research (NIPER), Sector 67, S.A.S. Nagar, Punjab-160062, INDIA

Phone: +91-172-229 2135

Fax: +91-172-221 4692

E-mail: ptiwari@niper.ac.in, pramiltiwari@yahoo.com

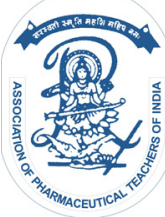

www.ijopp.org 
prise medications such as $1^{\text {st }}$ generation antihistamines, antipyretics or anti-inflammatory agents, cough suppressants (such as dextromethorphan) expectorants and decongestants (such as pseudoephedrine and phenylpropanolamine). ${ }^{4,5}$

While URTI are known to be common to the pediatric population, this study in north Indian children is the first one to describe the prescribing of drug(s) in children with URTI. This study was carried out to assess the utilization of drugs in children with URTI. An attempt has also been made to determine the incidence of URTI in different seasons.

\section{METHODOLOGY}

This year-long retrospective study was carried out at a pediatric outpatient setting. Out of 2981 screened paediatric patients, 1078 diagnosed with URTI were included in the study. The data was captured through an electronic prescribing package. The illnesses studied under URTI were - influenza like illness (ILI), acute otitis media (AOM), tonsillitis and URTI with bronchospasm.

The prescriptions of children up to 18 years of age approaching the clinic with diagnosis of URTI were studied. The information such as age, sex, weight, date of consultation, diagnosis, antibiotics and all other drugs prescribed and their doses, dosage form and route of administration, total duration of antibiotic and other drugs received, total dose of antimicrobial and other drugs administered were recorded. The data was organized using spreadsheet. It was analyzed for WHO recommended prescribing indicators.

In addition to the five WHO recommended prescribing indicators, the WHO recommended complementary indicators such as average cost of prescription and percentage of cost spent on the use of injections and antibiotics were also determined. ${ }^{6}$ In the computation of cost, only the direct cost of drugs in terms of maximum retail price (MRP) was considered. All the costs in the study were calculated in the Indian currency (INR) only $(1 \mathrm{INR}=0.01847 \mathrm{USD}=0.01415 \mathrm{EURO}=$ $0.01212 \mathrm{GBP})$. The prices as mentioned on the website of Current Index of Medical Specialities (http://www. mims.com/India) were used for computation of cost. All the computations for cost were done for the total duration of therapy.

The prescriptions were also analysed for morbidity pattern; utilization of different dosage forms, route of administration and antibiotics.

The patients were classified into five subgroups viz. new born (1day-1yrs), toddlers (1-3yrs), pre-schoolers (3-6yrs), schoolers (6-12yrs) \& adolescents (12-18yrs). The calendar year was classified into Spring (MarchMay), Summer (Jun-Aug), Autumn (Sept-Nov) and Winter (Dec-Feb).

All the indicators were represented as average \pm standard error of mean, median (Inter Quartile Range) or percentages, as applicable.

\section{RESULTS}

Of 2981 patients, only 1078 patients were diagnosed to have URTI. This consisted of 598 male \& 480 female patients.

Profile of patients: The average age and median age (IQR) were found to be $3.91 \pm 0.10 \mathrm{Y}$ and $3.1 \mathrm{Y}$ (1.3-5.3), respectively. One-third of the children diagnosed with URTI were toddlers $(34 \%)$. The preschoolers, schoolers, infants and adolescents contributed 30\%, 17\%, 16\% and $1 \%$, respectively.

Season wise prevalence of URTI: Maximum number of URTI cases were reported in winter season $(46 \%)$. The number of cases reported in autumn, spring and summer were very closely matching $(36 \%$, $33 \%$ and $31 \%$, respectively). It was found that the largest number of patients diagnosed with URTI were in the month of December while the least in the month of June. (Figure 1).

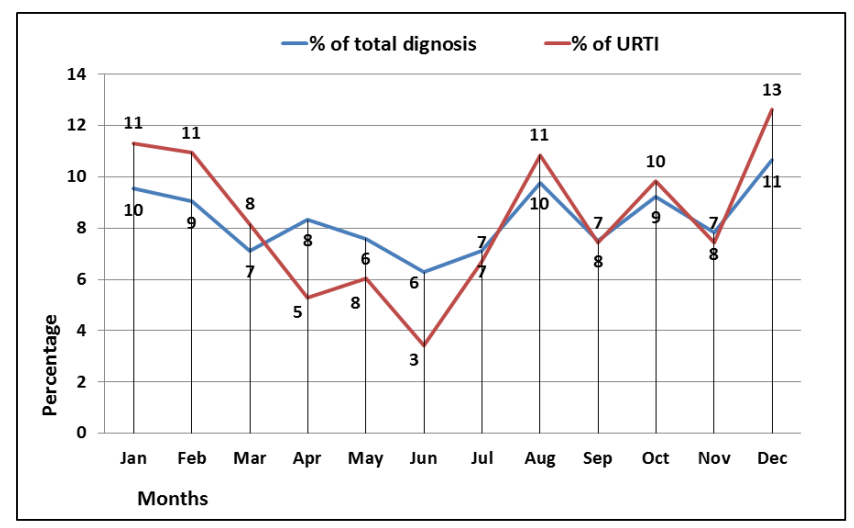

Figure 1: URTI patients over 12 months 
Profile of sign and symptoms observed in URTI patients: Fever was found to be be the most commonly observed in URTI patients (32\% of the patients) which was followed by cough $(24 \%)$, cold $(15 \%)$, throat congestion $(9 \%)$, cond sound $(9 \%)$, and nasal block $(6 \%)$.
Prescribing pattern: Among all the drugs utilized for the treatment of URTI, cough and cold combinations were found to be the most commonly utilized class of $\operatorname{drug}(27 \%)$. (Table 1)

\begin{tabular}{ccc}
\hline \multicolumn{3}{l}{ Table 1: Profile of different classes of drug prescribed } \\
\hline$\#$ & Class of drug & Number $(\%)$ \\
\hline 1 & Cold \& cough combinations & $751(27)$ \\
2 & Antipyretics & $582(21)$ \\
3 & Herbal cough/cold preparations & $503(18)$ \\
4 & Nasal drops & $420(15)$ \\
5 & Bronchodilators & $237(8)$ \\
6 & Antihistamines & $165(6)$ \\
7 & Antibiotics & $131(5)$ \\
\hline
\end{tabular}

Among antihistaminic drugs, cetrizine was found to be the maximally prescribed (64\% of the patients). Fixed-dose combination of paracteamol and ibuprofen was prescribed to $37 \%$ of the patients. (Table 2)
Cetrizine+ambroxol combination was found to be the most commonly prescribed combination (28\%) followed by combination of Chlorpheniramine+phenylephrine $(18 \%)$. (Figure 2)

\begin{tabular}{|c|c|c|}
\hline \# & Drug & Number (\%) \\
\hline & Antihistamines & \\
\hline 1 & Cetrizine & $106(64)$ \\
\hline 2 & Fexofenadine & $38(23)$ \\
\hline 3 & Loratadine & $14(8)$ \\
\hline \multirow[t]{2}{*}{4} & Promethazine & $7(4)$ \\
\hline & Antipyretics & \\
\hline 1 & $\mathrm{PCM}+\mathrm{IBU}$ & $218(37)$ \\
\hline 2 & PCM & $206(35)$ \\
\hline 3 & Mefenamic acid & $106(18)$ \\
\hline 4 & Mefenamic acid+Paracetamol & $52(9)$ \\
\hline
\end{tabular}

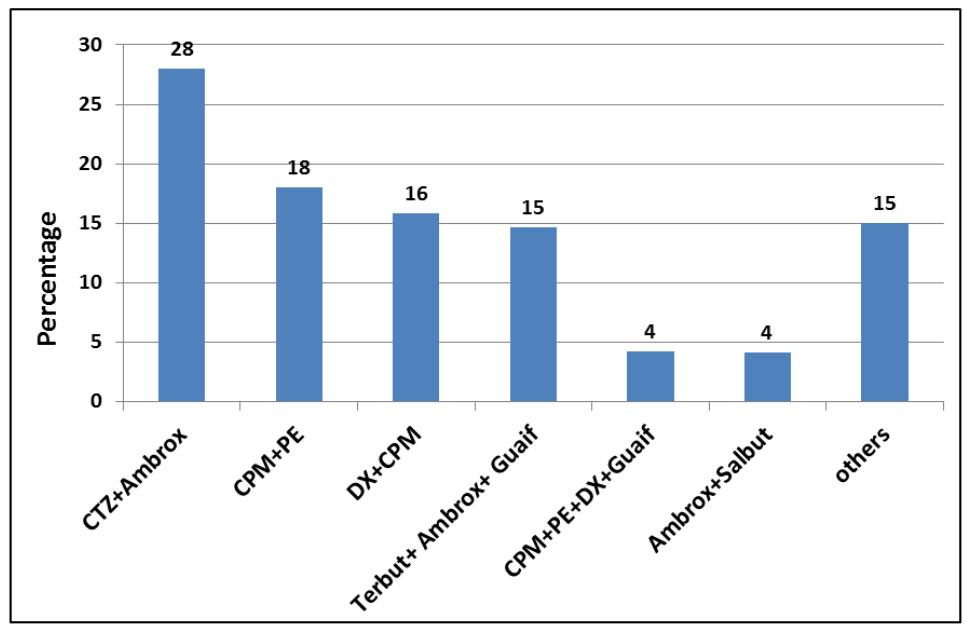

Figure 2: Cold and cough medicines prescribed

\#CTZ-cetrizine, Ambrox-ambroxol, PE-phenylephrine, CPM-chlorpheniramine, DX- dextromethorphan, Salbut-salbutamol, Bromhbromhexine, Guaif-guaifenesin, Others-Bromh+Guaif+Terbutaline, PE+PCM+CPM, PE+DX+triprolidine 
Pattern of antibiotic prescription: Antibiotics were prescribed to only $12 \%$ of the patients. Azithromycin was prescribed to the maximum number of patients followed by the prescribing of the combination of Amoxicillin and Clavulanic acid. (Figure 3)

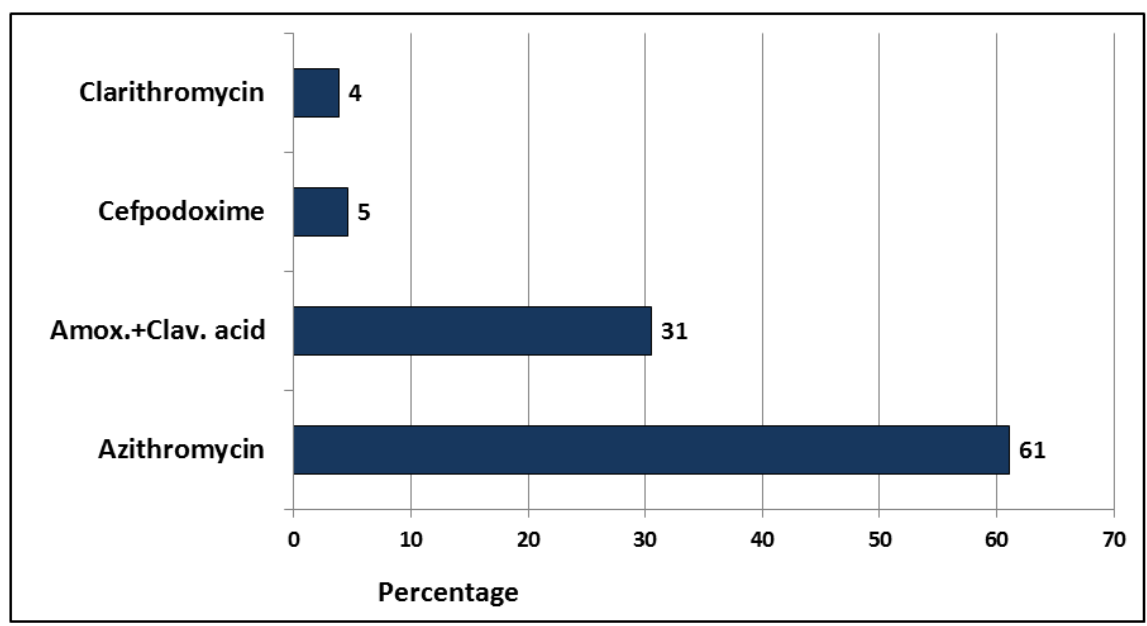

Figure 3: Antibiotic utilization in URTI

Prescribing Indicators: The average number of drugs per encounter was found to be 3.4 , with a minimum of 1 and maximum of 6 drugs. The proportion of patients receiving 2, 3, 4 and 5 drugs per prescription was found to be $21,37,30$ and $8 \%$, respectively. The median number of drugs per encounter was found to be 3 (IQR) (3-4).
Thirty eight percent of total 3292 drugs were prescribed from the NLEM-2011. Of all the drugs prescribed, only $7 \%$ were prescribed by generic name. Only 131 antibiotics were prescribed; and, this accounted for $12 \%$ of encounters with antibiotics in this study. It was found that none of the patient received a drug by the parenteral route. (Table 3 )

\begin{tabular}{lc} 
Table 3: WHO Prescribing indicators & Value \\
\hline Indicator & 3.4 \\
\hline Average number of drugs per encounter & $38 \%$ \\
Percentage of drugs prescribed from NLEM & $7 \%$ \\
Percentage of drugs prescribed by generic name & $0 \%$ \\
Percentage of encounters with an injection prescribed & $12 \%$ \\
\hline
\end{tabular}

Route of administration and dosage form: Out of the total drugs prescribed, $86 \%$ were administered by oral route. Nasal route of administration was the next most preferred route $(14 \%)$.

It was found that half of the drugs prescribed were syrups followed by the tablets (15\%), nasal drops (14\%), oral drops $(12 \%)$, and suspensions (10\%).

Pattern of FDC utilization: It was found out that a total of 1564 drugs i.e. $56 \%$ of the total drugs prescribed were as fixed dose combination (FDCs). Among FDCs, the prescribing of cough cold combination (48\%) were found to be maximum which was followed the prescribing of herbal cough and cold combination $(32 \%)$, antipyretic combination $(17 \%)$ and antibiotic combination $(3 \%)$. The number of drugs use in the fixed dose combinations were found to be 2, 3, 4, and $>4(61 \%, 4 \%, 3 \% \& 32 \%$ respectively).

Profile of Cost utilization in treatment of URTI: The average cost of drug treatment was found to be INR116.5 \pm 2.4 . The median cost of the treatment was INR98 (IQR of INR72-129). Nearly 11\% of the cost was spent on the antimicrobials. No cost was spent on the use of parenteral medication.

In case of cough and cold preparations, antibiotics, antihistamines and antiasthmatics the proportion of contribution of cost is higher than the proportion contributing of drug class from all the drugs prescribed. However, in case of antipyretics, herbal cough syrups and nasal drops the percentage of cost contribution is less than the contribution of drug class from overall drug prescribed. (Figure 4) 


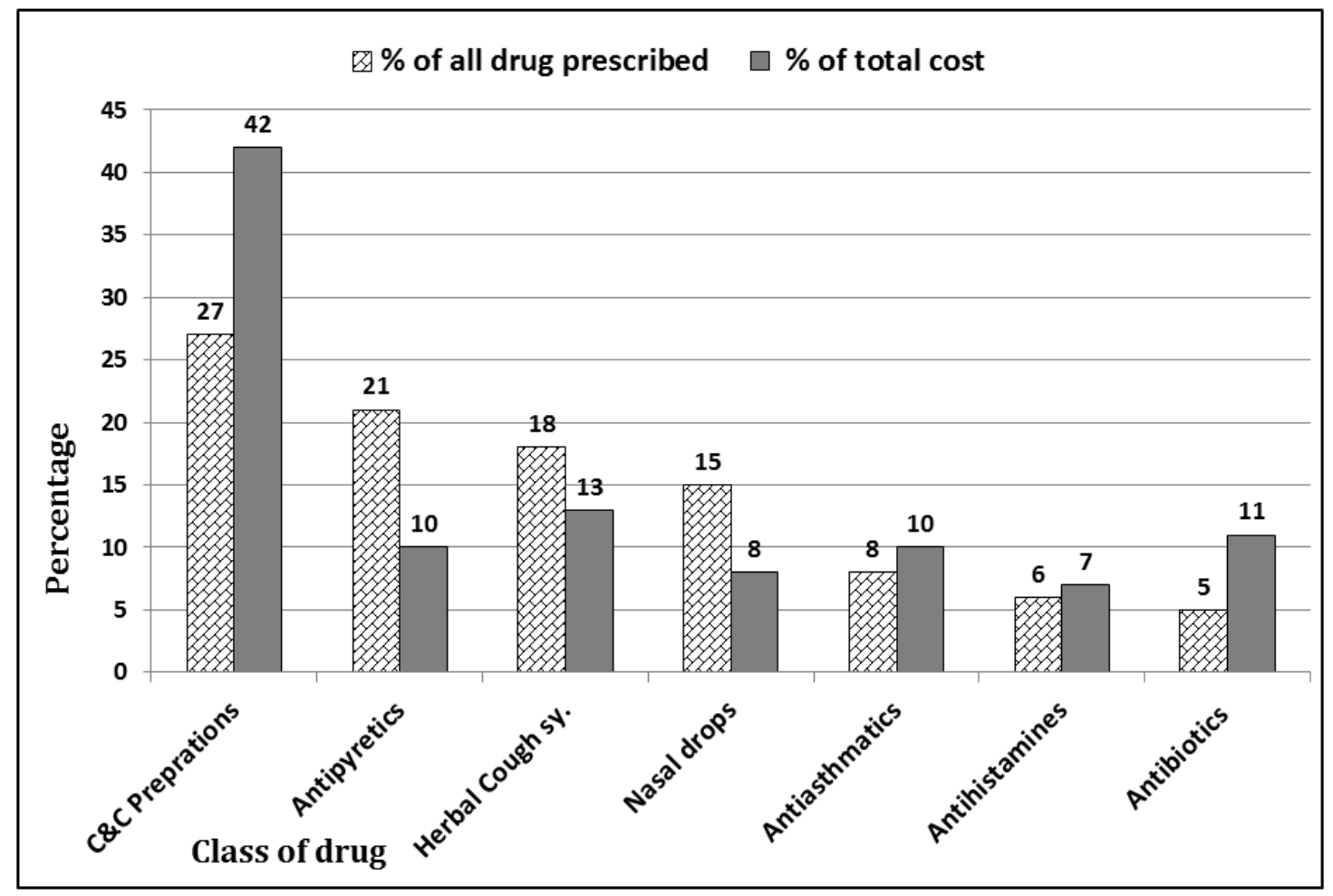

Figure 4: Comparison of cost spent vs contribution of drug class

\section{DISCUSSION}

The results of this 1-year long present study are based on the data obtained from 1078 patients diagnosed with URTI.

Over three-fourths of the patients with the diagnosis of upper respiratory tract infection were under 6 years of age $(80 \%)$. The findings are similar to the results of Sawalha et. al., from an inpatient setting, who have reported that $78 \%$ of the patients admitted with respiratory tract infection were less than 5 years in age. ${ }^{7} \mathrm{It}$ shows that children under age of 6 years are more susceptible to URTIs in comparison with other age groups.

In the present study, highest number of infections was reported in the winter. Highest cases were reported in month of March followed by August, January and February. Findlay et. al. have also demonstrated that Acute Respiratory Infection consultation rates displayed a seemingly bimodal annual pattern with the main peak in March and the secondary peak in September i.e., during the hot-dry and cold-dry seasons, respectively. The study carried out by Erling et. al. reported the prevalence of disease in temperate cold weather as $22.6 \%$. One of the probable reasons for higher occurrence of URTI in the winter is low humidity and increased dust, which may potentially damage immune barriers and 'carry' pathogens, respectively, further increasing the infection risk. ${ }^{8,9}$
It was found that $27 \%$ of the total medicines were cough and cold combination and only $5 \%$ of the total medicines were prescribed as antibiotics. The results of the study carried out by Das et. al. reported that $47 \%$ of the medicines were prescribed as cough and cold combination and $31 \%$ as antibiotics. ${ }^{10}$

The average number of drug prescribed increased with increase in number of diagnosis. In this study, average number of drugs prescribed was 3.4. The rational use of drugs demands to keep the number of drugs prescribed as low as possible to reduce the cost of treatment, drug interaction and adverse effects. The results of Das et. al. and Torvi et. al. from paediatric outpatient setting reported the average number of drugs prescribed as 2.37 and 2.22, respectively which is smaller than that in the present study. ${ }^{10,11}$ This difference could be due to the nature of the healthcare facility itself. The studies of Das et. al. and Torvi et. al. were conducted at a public OPD and at a tertiary care facility, respectively.

Thirty seven percent of the patients were prescribed with 3 drugs each. This has to be viewed with the fact that the most common diagnosis was URTI. For treatment of URTI, a combination of analgesic, cough syrups, saline drops are prescribed with or without antibiotics. ${ }^{3}$ This is comparable with the results of Tiwari et. al. in which three drugs were prescribed to $34 \%$ of patients. ${ }^{12}$

Indian Journal of Pharmacy Practice, Vol 7, Issue 2, Apr-Jun, 2014 
A very fair proportion of drugs were prescribed from the NLEM-2011 (38\%). Prescribing from the NLEM across India has been reported by Dimri et. al. and Shankar et. al. as $45 \%$ \& $68 \%$, respectively. ${ }^{13,14}$ The prescribing from NLEM in the later study was higher which was carried out at a public hospital setting. The prescribing from the NLEM should be promoted for optimal use of financial resources and to satisfy the health care needs of the majority of the population safely.

In the present study, only $7 \%$ of the medicines were prescribed by generic names. The study conducted by Mirza et. al. it as in southern India found as 30\%. ${ }^{15}$ The results of Mirza and co-workers are based on patient data from public hospital. However, the current study has been conducted in a private setting and this could have an influence on the generic prescribing. The possible reasons for nominal use of generic drugs could be prescribers' perception about the bioavailability and efficacy of generic formulations, prescribers' ignorance about the price variations between generic and branded and the lack of information on the availability of various generic formulations. A second possible reason could be that the branded drugs are easily available and names are easy to recall for the prescriber and dispenser.

The antibiotics encountered in URTIs were nearly $12 \%$ which is far smaller than $52 \%$ reported by Senok et. al and $60 \%$ by Ayranci et. al. ${ }^{16,17}$ This is a reflection of the critical decision in medical care while prescribing the antibiotics for URTI. In present study, macrolides were the most commonly prescribed antibiotic followed by Penicillin (31\% \& 65\%). Mazzaglia et. al. have also confirmed macrolides as the commonly prescribed class of antibiotic. $^{18}$

Khan et. al. and Ain et. al. have shown the use of $\beta$-lactam antibiotics to the tune of $74 \%$ and $45 \% .^{19,20}$ According to the National Institute of Health (NIH) pediatric treatment guideline for respiratory tract infection, penicillin is the first line antibiotic for pharyngitis; and, macrolide is the drug of choice for treating cough and bronchitis. ${ }^{3}$ The result of present study had demonstrated that penicillins and macrolides were the most commonly utilized antibiotic used for the treatment of URTI.

In this study, the average cost of drugs per encounter for treating URTI was found to be INR116 which is close to USD2; ( $1 \mathrm{INR}=0.02 \mathrm{USD})$. The average cost of treating URTI, in a Canadian analysis carried out by Wang et. al., was USD $13 .{ }^{21}$ In the current study, the cost of the drugs in the prescription was very reasonable. The cost of prescription increases as the number of injection and antimicrobial drug in the prescription increase. In this study, no parenteral medication was used.
In comparison to the results of Mungrue et. al., the results of this study confirm that only $21 \%$ of all drugs prescribed were analgesic/antipyretic. ${ }^{22}$ Ibuprofen+Paractamol combination was the most commonly prescribed drug as Ibuprofen+Paractamol combination was more effective in relieving fever. Ibuprofen, Paracetamol and Mefenamic acid are the drug of choice for the fever in children. Paracetamol alone and in combination were the most prescribed drug in the study as Paracetamol is a proven and safe antipyretic. Likewise, Antihistamines to the tune of only $6 \%$ of the total number of drug prescribed was found smaller than the results of Jhaj et. al. in which antihistamine were prescribed as $36 \%$ of the total number of drug prescribed. ${ }^{23}$ Antihistamines are among the most commonly prescribed medicines in pediatric practice. ${ }^{24}$ Formulations containing antihistamines only, or in combination with other drugs (antitussive agents, systemic decongesting drugs, etc.) are widely used for control of symptoms and the treatment of upper respiratory tract infections in children. ${ }^{25}$

Oral route is the most convenient and safest route of drug administration among children. The most commonly used dosage form was syrup $(60 \%)$. The convenience of oral dosage form as the easiest to administer in the children in outpatient setting explains this. The result was found comparable to the study carried out by Karande et. al. in which $61 \%$ of the drugs were prescribed in the form of syrup. ${ }^{26}$

In present study, $56 \%$ of the total drugs were prescribed as FDCs. Das et. al. and Jhaz et. al. have reported it to be $60 \%$ each. ${ }^{10,23}$ Among fixed dose combinations, two drug combinations was the most commonly prescribed drug. antipyretic + decongestant, decongestant + antihistamine, antihistamine+antipyretic combinations were the most commonly prescribed drugs.

In URTI treatment, use of antibiotic is always an area of concern. As there was no culture report available in outpatient setting, whether to prescribe an antibiotic or not is physician's decision. There is a scope of improvement in prescribing from NLEM-2011 and prescribing by generic name of drugs.

Irrational (Misuse/overuse) of Antibiotics is an important public health issue that affects the community and the individual. Use of antibiotic in children for the treatment of upper respiratory tract infections is evidently inappropriate unless the infection was proven to be bacterial. The misuse of antibiotic, especially in children can leads to bacterial resistance and various undesirable side effects associated with the antibiotic use. In present study, a large number of patients were treated without the use of antibiotic, which is a good sign of 
rational prescribing. The minimal antibiotic and injections usage reflects judicious choice on the part of the prescriber. The present study helps in building evidence on the rational use of antibiotic in upper respiratory tract infection in a pediatric outpatient clinic.

\section{REFERENCES}

1. Feld LG, Hyams JS. Respiratory Tract Infections in Children: New Developments in Diagnosis and Treatment. Consensus in Pediatrics. 2009; 1 (11): 1-46.

2. Cotton MF, Innes S, Jaspan H, Madide A, Rabie H. Management of upper respiratory tract infections in children. SA Fam Pract. 2008; 50 (2): 6-12.

3. NICE. Respiratory tract infections - antibiotic prescribing Prescribing of antibiotics for self-limiting respiratory tract infections in adults and children in primary care. National Institute of Health and Clinical Excellenece. 2008; 2008. p.1-122.

4. Simasek M, Blandino DA. Treatment of the common cold. Am Fam Physician. 2007; 75 (4): 515-20.

5. Arroll B. Common cold. Clinical Evidence. 2008; 06:1510.

6. Bimo H, Hogerzeil V, Choudhury A, Das A, Diwan V, Kafle K, et al. How to Investigate Drug Use in Health Facilities: Selected Drug Use Indicators - EDM Research Series No. 007. World Health Organization. 1993. 1-88.

7. Sawalha A, Al-Bishtawi G, Al-Khayyat L, Sweileh W, Al-Ramahi R, Jaradat N. Pattern of Parenteral Antimicrobial Prescription among Pediatric â Patients in Al-Watani Governmental Hospital in Palestine. An-Najah University Journal for Research. 2006; 20: 191-206.

8. Findley SE, Medina DC, Sogoba N, Guindo B, Doumbia S. Seasonality of childhood infectious diseases in Niono, Mali. Glob Public Health. 2010; 5 (4): 381-94.

9. Erling V, Jalil F, Hanson LA, Zaman S. The impact of climate on the prevalence of respiratory tract infections in early childhood in Lahore, Pakistan. J Public Health Med. 1999; 21 (3): 331-9.

10. Das B, Sarkar C, Majumder AG. Medication use for pediatric upper respiratory tract infections. Fundam Clin Pharmacol. 2006; 20 (4): 385-90.

11. Torvi JR, Dambal S. Drug prescription pattern in paediatric out patient clinic in a tertiary hospital. Curr Pediatr Res. 2011; 15 (2): 77-80.

12. Tiwari $P$, Ahlawat $R$, Gupta $G$. Pattern of prescribing at a paediatric outpatient setting in northern India. Ind J Pharm Pract. 2012; 5 (1): 40-4.

13. Dimri S, Tiwari P, Basu S, Parmar VR. Drug use pattern in children at a teaching hospital. Indian Pediatrics. 2009; 46 (2): 165.
14. Shankar PR, Upadhyay DK, Subish P, Dubey AK, Mishra P. Prescribing patterns among paediatric inpatients in a teaching hospital in western Nepal. Singapore medical journal. 2006; 47 (4): 261.

15. Mirza NY, Desai S, Ganguly B. Prescribing pattern in a pediatric out-patient department in Gujarat. Bangladesh Journal of Pharmacology. 2008; 4 (1): 39-42.

16. Ayranci U, Akgun Y, Unluoglu I, Kiremitci A. Antibiotic prescribing patterns for sore throat infections in a university-based primary care clinic. Ann Saudi Med. 2005; 25 (1): 22-8.

17. Senok AC, Ismaeel AY, Al-Qashar FA, Agab WA. Pattern of upper respiratory tract infections and physicians' antibiotic prescribing practices in Bahrain. Med Princ Pract. 2009; 18 (3): 170-4.

18. Mazzaglia G, Greco S, Lando C, Cucinotta G, Caputi A. Adult acute upper respiratory tract infections in Sicily: pattern of antibiotic drug prescription in primary care. Journal of Antimicrobial Chemotherapy. 1998; 41 (2): 259-66.

19. Ain MR, Shahzad N, Aqil M, Alam MS, Khanam R. Drug utilization pattern of antibacterials used in ear, nose and throat outpatient and inpatient departments of a university hospital at New Delhi, India. J Pharm Bioallied Sci. 2010; 2 (1): 8-12.

20. Khan FA, Nizamuddin S, Salman MT. Patterns of prescription of antimicrobial agents in the Department of Otorhinolaryngology in a tertiary care teaching hospital. African Journal of Pharmacy and Pharmacology. 2011; 5 (14): 1732-38

21. Wang EEL, Einarson T, Kellner JD, Conly J. Antibiotic (ABX) Prescribing for Respiratory Tact Infections in Preschool Children: Analysis of the Saskatchewan Drug Database [dagger] 550. Pediatric research. 1998; 43 (S4): 96.

22. Mungrue K, Brown T, Hayes I, Ramroop S, Thurston P, Pereira LP. Drugs in upper respiratory tract infections in paediatric patients in North Trinidad. Pharmacy Practice (Internet). 2009; 7 (1).

23. Jhaj R, Bhargava VK, Uppal R, Lekha S, Reetfia K, Kaur N. Use of cold medications for upper respiratory tract infections in children. Pharmacoepidemiol Drug Saf. 2001;10 (4): 323-7.

24. Vernacchio L, Kelly JP, Kaufman DW, Mitchell AA. Cough and cold medication use by US children, 1999-2006: results from the slone survey. Pediatrics. 2008; 122 (2): 2008-0498.

25. Sutter Al, Lemiengre M, Campbell H, Mackinnon HF. Antihistamines for the common cold. Cochrane Database Syst Rev. 2003; (3): CD001267.

26. Karande S, Sankhe P, Kulkarni M. Patterns of prescription and drug dispensing. Indian J Pediatr. 2005; 72 (2): 117-21. 